\title{
471832 - POST- OPERATIVE VASO VAGAL SYNCOPE: IS INTRATHECAL ANESTHESIA THE CAUSE?
}

\author{
Tania Di Renna, MD, John Penning, MD, FRCP, Gregory Bryson, MD \\ University of Ottawa, Ottawa, ON, Canada
}

Introduction: A multimodal analgesia protocol (MMAP) for prostatectomy patients, involving pre operative oral NSAIDs, a spinal anesthetic with 4-6 mls of 0.5\% bupivicaine with 200 - 400 ug intrathecal morphine combined with a general anesthetic, has been followed for several years at our institution. Post operatively patients receive NSAIDs for 5 days, straight Tylenol and prn oral morphine. Nurses report that frank vaso vagal syncope or presyncopal symptoms have been a common occurrence in prostatectomy patients since this MMAP has been in effect. The purpose of our study was to determine if the intrathecal anesthetic is contributing to the post operative syncope or presyncope symptoms.

Methods: Following ethics board approval, we identified 148 consecutive patients undergoing radical prostatectomy who received intrathecal anesthesia between 20032005. As a control population we also identified 31 patients who underwent radical prostatectomy and did not receive an intrathecal anesthetic. The data collected included patients' demographics, duration of surgery, intraoperative blood loss, intravenous fluids, dose of intrathecal anesthetic and level of block in the PACU. Our outcome measures were the number of syncopal or presyncopal events, peak VAS scores recorded on post operative day one and length of stay. We analyzed our data with one way ANOVA and post hoc tests.

Results: Of the 148 prostatectomy patients who received intrathecal anesthetics, 43 were found to have either presyncopal symptoms or frank syncope the morning of the first post operative day. In contrast only 1 of the 31 prostatectomy patients who did not receive intrathecal analgesia had a presyncopal episode. The difference between age, ASA scores, blood loss, intraoperative intravenous fluids and length of stay, when compared between the two groups, was not statistically significant. There was, however, a significant difference between intraoperative vasopressor use, syncopal events and post operative pain scores.

Discussion: Our study indicates that intrathecal anesthetics in prostatectomy patients, although a superior modality for pain control, may contribute to post operative syncope or presyncope. We propose that a residual sympathetic blockade from large doses of intrathecal bupivicaine may be key producing in (pre)syncope.

References: Preemptive Epidural Analgesia and Recovery From Radical Prostatectomy A Randomized Controlled Trial Allan Gottschalk et al., JAMA. 1998;279:1076-1082.

Results 


\begin{tabular}{|c|c|c|c|}
\hline Parameter & Spinal + GA & GA only & P value \\
\hline N & 148 & 31 & \\
\hline Age & $61+/-6.5$ & $60+/-6.5$ & 0.56 \\
\hline Duration of OR (hrs) & $4.15+/-0.4$ & $3.8+/-0.4$ & 0.10 \\
\hline Estimated Blood Loss (mL) & $730+/-469$ & $760+/-516$ & 0.14 \\
\hline Crystalloid (mL) & $3324+/-1014$ & $3200+/-610$ & 0.5 \\
\hline Colloid & $476+/-373$ & $375+/-279$ & 0.2 \\
\hline Phenylephrine infusion (\%) & 34 & 0 & 0.00 \\
\hline Total Events (\%) & 28 & 3 & 0.00 \\
\hline Syncope & 9 & 0 & \\
\hline Presyncope & 19 & 3 & 0.44 \\
\hline Decrease in Hemoglobin (g/L) & $40+/-8.2$ & $41+/-10$ & 0.00 \\
\hline Highest VAPS @ POD 1 & $2+/-1$ & $4+/-3$ & 0.00 \\
\hline 24 IV Morphine consumption (mg) & $2.5+/-4$ & $10+/-4$ & 0.95 \\
\hline Length of Stay (days) & $4+/-2$ & $4+/-1$ & \\
\hline
\end{tabular}

